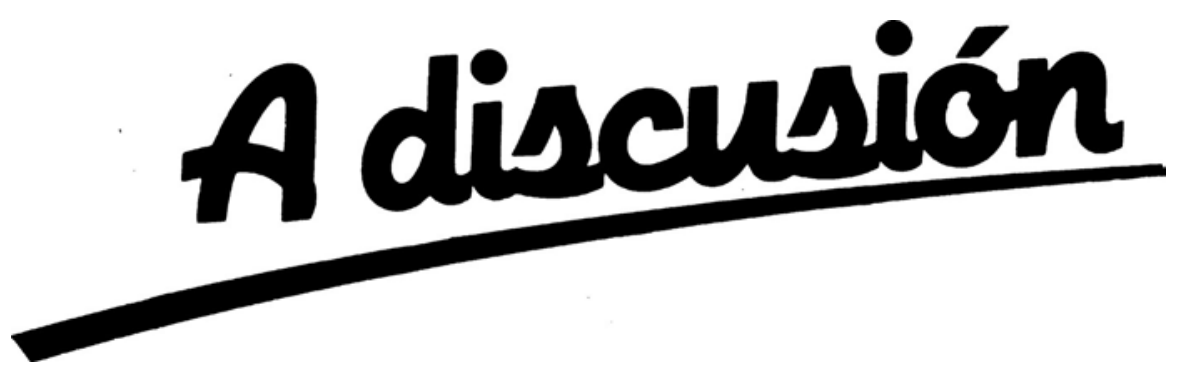

\title{
THE NEOCLASSICAL GROWTH MODEL WITH HETEROGENEOUS QUASI-GEOMETRIC CONSUMERS*
}

\section{Lilia Maliar and Serguei Maliar**}

\author{
WP-AD 2003-25
}

Corresponding author: Lilia Maliar, Universidad de Alicante. Departamento de Fundamentos del Análisis Económico. Carretera de San Vicente del Raspeig s/n, San Vicente del Raspeig, 03080, Alicante, Spain. e-mail: maliarl@merlin.fae.ua.es.

Editor: Instituto Valenciano de Investigaciones Económicas, S.A.

Primera Edición Julio 2003

Depósito Legal: V-3471-2003

IVIE working papers offer in advance the results of economic research under way in order to encourage a discussion process before sending them to scientific journals for their final publication.

\footnotetext{
* We thank seminar participants at SED 2003 (Paris) for comments on the earlier version of this paper entitled "Time-inconsistent preferences in a heterogeneous agents world". This research was partially supported by the Instituto Valenciano de Investigaciones Económicas and the Ministerio de Ciencia y Tecnología de España, BEC 2001-0535.
}

** L. \& S. Maliar: Departamento de Fundamentos del Análisis Económico, Universidad de Alicante. 


\title{
THE NEOGLASSICAL GROWTH MODEL WITH HETEROGENEOUS QUASI-GEOMETRIC CONSUMERS

\author{
Lilia Maliar and Serguei Maliar
}

\begin{abstract}
This paper studies how the assumption of quasi-geometric (quasihyperbolic) discounting affects the individual consumption-savings behavior in the context of the standard one-sector neoclassical growth model with heterogeneous agents. The agents are subject to idiosyncratic shocks and face borrowing constraints. We confine attention to an interior Markov recursive equilibrium. The consequence of quasi-geometric discounting is that the effective discount factor of an agent is not a constant, but an endogenous variable which depends on the agent's current state. We show, both analytically and by simulation, that this feature of the model can significantly affect its distributional implications.
\end{abstract}

JEL Classification: D91, E21, G11

Keywords: time inconsistency, quasi-geometric discounting, quasi-hyperbolic discounting, idiosyncratic shocks, wealth inequality. 


\section{Introduction}

Quasi-geometric (quasi-hyperbolic) discounting is a specific form of timeinconsistency in preferences when the discount factor, applied between today and tomorrow, is different from the one, used on all dates advanced further in the future. The first studies on quasi-geometric discounting date back to Strotz (1955-1956), Pollak (1968), and Phelps and Pollak (1968), although interest in this subject has revived recently, e.g., Laibson (1997), Laibson, Repetto and Tobacman (1998), Barro (1999), Harris and Laibson (2001), Krusell and Smith (2000, 2003), Krusell, Kuruşçu and Smith (2002), Luttmer and Mariotti (2002).

This paper studies the quantitative implications of a heterogeneous-agent general-equilibrium extension of Harris and Laibson's (2001) model. We specifically consider a version of the standard one-sector neoclassical growth model with a continuum of quasi-geometric agents who are subject to idiosyncratic labor productivity shocks and who face borrowing constraints. We confine attention to an interior Markov recursive solution to the individual utility maximization problem. We focus on a stationary equilibrium such that the prices of capital and labor are consistent with the optimal decisions of agents, as in Aiyagari (1994).

With the assumption of quasi-geometric discounting, the effective discount factor of an agent is not a constant, but an endogenous variable which depends on the agent's current state. In particular, if the consumption function is strictly concave, then the effective discount factor of the short-run impatient agent is increasing in wealth. As a result, the rich are more patient than the poor, so that the model with quasi-geometric short-run impatient agents produces a larger dispersion of wealth than the standard setup where the rich and the poor are equally patient. This implication is of interest given that the standard model with a constant discount factor dramatically underpredicts the size of wealth inequality relative to the data, e.g., Quadrini and Rios-Rull (1997).

In a calibrated version of the model, we find that the effects associated with the assumption of quasi-geometric discounting are quantitatively significant. For example, in our benchmark model with short-run impatient agents, the wealth holdings of the bottom $40 \%$ of the population decline by $29 \%$, the 
wealth holdings of the top $1 \%$ increase by $13 \%$, and the Gini coefficient of the wealth distribution increases by $18 \%$ compared to the standard geometricdiscounting setup. These improvements are however too small for the model to reproduce the size of wealth inequality observed in the data. Furthermore, we find that, in our general equilibrium model, the size of precautionary savings is not substantially affected by the presence of quasi-geometric discounting. This is contrary to what Laibson, Repetto and Tobacman (1998) have obtained in a partial equilibrium setup.

The rest of the paper is organized as follows. Section 2 formulates the model, derives the optimality conditions and discusses some of the model's implications. Section 3 describes the methodology of the quantitative study and presents the results from simulations, and finally, Section 4 concludes.

\section{The model}

Time is discrete and the horizon is infinite, $t \in T$, where $T=\{0,1,2, \ldots\}$. The economy is populated by a continuum of infinitely-lived agents with names on a closed interval $[0,1]$. The agents inelastically supply their total time endowment (equal to one) to the market. The labor productivities of the agents are subject to idiosyncratic shocks. The shocks follow a firstorder Markov process and are uncorrelated across the agents. All possible realizations of productivity shocks are in the set $\mathcal{S}=\left[s_{\min }, s_{\max }\right] \subset R_{+}$. At each point of time, the agents also differ in asset holdings, which somehow summarize information on past realizations of shocks. Assets are restricted to be in the set $\mathcal{A}=[-b, \infty) \subset R$. That is, the agents are only allowed to borrow up to a certain limit $b$.

In every period $t \in T$, an agent seeks to maximize the expected present value of the sum of one-period utilities from $t$ forward by choosing an optimal path for consumption. The agent discounts the future by using the quasigeometric weights. Specifically, in period $t$, the agent puts the weight 1 on the utility of period $t$ and the weight $\beta \delta^{\tau+1-t}$ on the utility of each period $\tau>t$, where the discounting parameters $\beta$ and $\delta$ are such that $\beta>0$ and $0<\delta<1$. Consequently, at each date $t \in T$, the agent solves the following problem

$$
\max _{\left\{c_{\tau}, a_{\tau+1}\right\}_{\tau=t}^{\infty}}\left\{u\left(c_{t}\right)+E_{t} \sum_{\tau=t}^{\infty} \beta \delta^{\tau+1-t} u\left(c_{\tau+1}\right)\right\}
$$


subject to

$$
\begin{gathered}
c_{\tau}+a_{\tau+1}=w s_{\tau}+(1+r) a_{\tau}, \\
a_{\tau+1} \geq-b,
\end{gathered}
$$

where initial condition $\left(a_{t}, s_{t}\right)$ is given. Here, $c_{\tau}, a_{\tau}$ and $s_{\tau}$ are consumption, asset holdings and the labor productivity shock, respectively; $r$ is the interest rate; $w$ is the wage per unit of efficiency labor; $E_{\tau}$ is the expectation, conditional on all information about the agent's idiosyncratic shocks available at $\tau$. The momentary utility function $u(c)$ is continuously differentiable, strictly increasing, strictly concave and satisfies $\lim _{c \rightarrow 0} u^{\prime}(c)=\infty{ }^{1}$

If $\beta<1$, the short-run discount factor $\beta \delta$ is lower than the long-run discount factor $\delta$. The agent plans to save much in the future, however, as the future comes around, she changes her mind and saves less than she would have originally committed to if commitment was available. This case is often referred to in the literature, as quasi-hyperbolic discounting, because of a qualitative similarity with the case, where the discount factor is given by an increasing-over-time generalized hyperbolic function $(1+\alpha t)^{-\nu / \alpha}$, with $\alpha, \nu>0$. If $\beta>1$, then the situation is reversed: the agent consumes less in the future than she would have committed to. As argued in Hall (1998), both alternatives, $\beta<1$ and $\beta>1$, are plausible. Following Krusell and Smith (2000), we will refer to both cases, $\beta<1$ and $\beta>1$, as quasi-geometric discounting: except for the current date, the weights on momentary utility functions decline geometrically.

As argued in the literature, e.g., Laibson (1997), one can view a quasigeometric consumer in different periods as a collection of temporal selves, who play an infinite-horizon game. Each self $t$ has the preferences defined over the stream of consumption $\left\{c_{\tau}\right\}_{\tau=t}^{\infty}$ and solves the problem (1) - (3). We focus on the case when self $t$ only has a direct control over the current consumption, $c_{t}$, i.e., the agent cannot commit herself to future actions.

The production side of the economy consists of the representative firm. Given the factor prices $r$ and $w$, the firm rents capital $K_{t}$ and hires labor $N_{t}$ to maximize period-by-period profits. The technology is described by $F\left(K_{t}, N_{t}\right)+(1-d) K_{t}$. The production function $F$ has constant returns to

\footnotetext{
${ }^{1}$ We do not impose the nonnegativity of consumption explicitly because it is guaranteed by the assumption $\lim _{c \rightarrow 0} u^{\prime}(c)=\infty$.
} 
scale, is strictly increasing, strictly concave, continuously differentiable and satisfies the appropriate Inada conditions. The depreciation rate of capital is $d \in(0,1]$.

\subsection{Recursive formulation and the Euler equation}

As shown in Harris and Laibson (2001), the problem (1) - (3) can be written recursively. To be specific, let us assume that in all periods, the agent decides on consumption according to the same consumption function, $c_{t}=C\left(a_{t}, s_{t}\right)$. Then, without time subscripts, we have the following recursive formulation:

$$
W(a, s)=\max _{c}\left\{u(c)+\beta \delta E\left[V\left(a^{\prime}, s^{\prime}\right) \mid s\right]\right\},
$$

where given $(a, s)$, the value function $V$ solves the functional equation

$$
V(a, s)=u[C(a, s)]+\delta E\left\{V\left[w s+(1+r) a-C(a, s) ; s^{\prime}\right] \mid s\right\}
$$

subject to the budget constraint

$$
a^{\prime}=w s+(1+r) a-c
$$

and the borrowing constraint

$$
a^{\prime} \geq-b
$$

The problem (4) - (7) is to be solved for the unknown value functions $W(a, s)$, $V(a, s)$ and the consumption function $C(a, s)$. We assume that the above functions are continuous and differentiable. These assumptions will be in force throughout the remainder of the paper.

The value functions $W\left(a_{t}, s_{t}\right)$ and $V\left(a_{t}, s_{t}\right)$ show how the agent values the assets $a$ in periods $t$ and $t-1$, respectively. Under standard geometric discounting $(\beta=1)$, the value of $a_{t}$ in period $t$ is the same as in period $t-1$, so that $W\left(a_{t}, s_{t}\right)$ and $V\left(a_{t}, s_{t}\right)$ coincide. Under quasi-geometric discounting $(\beta \neq 1)$, the values of the assets $a_{t}$ at $t$ and $t-1$ are not equal and neither are $W\left(a_{t}, s_{t}\right)$ and $V\left(a_{t}, s_{t}\right)$. This is precisely what time inconsistency means.

If the problem $(4)-(7)$ has an interior solution, then such a solution satisfies the quasi-geometric Euler equation:

$$
u^{\prime}\left(c_{t}\right) \geq \delta E_{t}\left\{u^{\prime}\left(c_{t+1}\right)\left[1+r-(1-\beta) \cdot C_{1}\left(a_{t+1}, s_{t+1}\right)\right]\right\},
$$


where $u^{\prime}$ is the derivative of the utility function $u$, and $C_{1}$ is the first-order partial derivative of the consumption function $C$ with respect to the first argument. The Euler equation holds with strict inequality if the borrowing limit is reached. We assume that the solution to the Euler equation exists and is unique. ${ }^{2}$

\subsection{Equilibrium}

Let $x$ be a probability measure defined on $\mathcal{B}$, where $\mathcal{B}$ denotes the Borel subset of the set of all possible individual states $\mathcal{A} \times \mathcal{S}$. For all $B \in \mathcal{B}, x_{t}(B)$ is the mass of agents whose individual states lie in $B$ at time $t$. Given that $x_{t}$ is a probability measure, the total mass of agents is equal to 1 .

Denote by $P(a, s, B)$ the conditional probability that an agent with state $(a, s)$ will have an individual state lying in set $B$ in the next period. The function $P$ is defined as

$$
P(a, s, B)=\operatorname{Prob}\left(\left\{s^{\prime} \in \mathcal{S}:\left[A(a, s), s^{\prime}\right] \in B\right\} \mid s\right),
$$

where $A(a, s) \equiv a^{\prime}=w s+(1+r) a-C(a, s)$ is the decision function for assets (the asset function). Then, the law of motion of $x_{t}$ is: $x_{t+1}(B)=$ $\int_{\mathcal{A} \times \mathcal{S}} P(a, s, B) d x_{t}$ for all $t \in T$ and all $B \in \mathcal{B}$.

Labor and capital inputs are given by the sum of efficiency units of labor supplied by all workers, $N_{t}=\int_{\mathcal{A} \times \mathcal{S}} s_{t} d x_{t}$, and the sum of assets held by all agents, $K_{t}=\int_{\mathcal{A} \times \mathcal{S}} a_{t} d x_{t}$, respectively.

The fact that there is a continuum of agents guarantees that the mass of agents with the shock $s$ at $t$ and the shock $s^{\prime}$ at $t-1$ is equal to the conditional probability, $\operatorname{Prob}\left(s \mid s^{\prime}\right)$. Since labor productivity shock follows a first-order Markov process, such probability depends only on the recent past and is the same in all periods. Hence, $N_{t}$ is a constant. For convenience, we normalize it to one, $N=1$.

We only study such equilibria in which the period- $t+1$ probability measure $x_{t+1}$ is the same as the period- $t$ probability measure $x_{t}$, for all $t \in T$.

\footnotetext{
${ }^{2}$ It has been shown in the literature that the assumption of quasi-geometric discounting can lead to non-monotonic or discontinuous decision rules (Harris and Laibson, 2001) and to multiple equilibria (Krusell and Smith, 2000). It appears however that the interior equilibrium (the one satisfying the Euler equation) is unique (see Krusell, Kuruşçu and Smith, 2002, for a discussion).
} 
In this case, we say that the probability measure is stationary and denote it by $x^{*}$. The stationarity of $x^{*}$ implies that the aggregate capital stock is constant, $K=\int_{\mathcal{A} \times \mathcal{S}} a_{t} d x^{*}$ for all $t \in T$ (even though the assets of each agent vary stochastically over time).

Definition. A stationary equilibrium is defined as a stationary probability measure $x^{*}$, an optimal consumption function $C(a, s)$, and positive real numbers $(K, r, w)$ such that

(1) $x^{*}$ satisfies $x^{*}=\int_{\mathcal{A} \times \mathcal{S}} P(a, s, B) d x^{*}$ for all $B \in \mathcal{B}$;

(2) $C(a, s)$ solves the Euler equation (8) for a given pair of prices $(r, w)$;

(3) $(r, w)$ are such that the firm's profit is maximum

$$
r=F_{1}(K, 1)-d \quad w=F_{2}(K, 1),
$$

where $F_{1}$ and $F_{2}$ are the first-order partial derivatives of the production function $F$ with respect to capital and labor inputs, respectively;

(4) $K$ is the average of the agents' decisions: $K=\int_{\mathcal{A} \times \mathcal{S}} A(a, s) d x^{*}$.

\subsection{Model's implications}

Under the assumption of standard geometric discounting, $(\beta=1)$, the discount factor is a constant, equal to $\delta$ in all periods. However, if discounting is quasi-geometric, $(\beta \neq 1)$, the effective discount factor is an endogenous variable, which depends on the state of the agent. In this section, we illustrate some properties of such an endogenous discount factor. We employ a simplifying assumption that the borrowing limit is never reached. ${ }^{3}$

In the absence of borrowing restrictions, the quasi-geometric Euler equation holds with equality and can be re-written as

$$
u^{\prime}\left(c_{t}\right)=\delta_{t+1}(1+r) E_{t}\left[u^{\prime}\left(c_{t+1}\right)\right],
$$

where $\delta_{t+1}$ is an "effective discount factor", defined as

$$
\delta_{t+1} \equiv \delta_{t+1}\left(a_{t+1}, s_{t+1}\right)=\delta \cdot\left[1-\frac{1-\beta}{1+r} \cdot \frac{E_{t}\left[u^{\prime}\left(c_{t+1}\right) C_{1}\left(a_{t+1}, s_{t+1}\right)\right]}{E_{t}\left[u^{\prime}\left(c_{t+1}\right)\right]}\right]
$$

\footnotetext{
${ }^{3}$ At the point where the borrowing constraint begins to bind, the consumption function has a kink and, therefore, is not continuously differentiable.
} 
If $\beta=1$, then $\delta_{t+1}=\delta$ for all $t \in T$ and condition (9) reduces to the standard Euler equation. Before we characterize the properties of $\delta_{t+1}$ under $\beta \neq 1$, let us establish one useful result regarding the consumption function.

Lemma $1 C(a, s)$ is strictly increasing in a for all $a \in \mathcal{A}, s \in \mathrm{S}$.

Proof. See appendix A. \|

The proof of Lemma 1 relies on the assumption that a solution to the Euler equation (8) exists, it is unique and that the value function $W$ is continuously differentiable. All of these properties were satisfied in our simulations, when $\beta$ was sufficiently close to one. In general, the properties of the solution to the studied model are not known. ${ }^{4}$

The implication of this result for the discount factor $\delta_{t+1}$ is as follows:

Proposition 1 If $\beta \lesseqgtr 1$, then $\delta_{t+1} \lesseqgtr \delta$ for all $a \in \mathcal{A}, s \in \mathrm{S}$.

Proof. Under the assumption that $u^{\prime}$ is strictly increasing and with the result of Lemma 1, the proof of Proposition 1 follows from (10) directly. \|

Consider first the case $\beta<1$. Proposition 1 shows that under such quasi-geometric discounting, the agent has the discount factor $\delta_{t+1}<\delta$ and therefore, is less patient than that with $\beta=1$. Precisely what determines the patience of the agent? Two factors are relevant here. First, self $t$ is impatient $\left(\delta_{t+1}<\delta\right)$, because she is the short-run impatient, i.e., she has the short-run discount factor $\beta \delta$, which is lower than the long-run discount factor $\delta$. Second, self $t$ is impatient because the subsequent self $t+1$ is the short-run impatient. To see the point, consider the first-order condition of the problem $(4)-(7)$ with respect to consumption. Self $t+1$ 's choice of $c_{t+1}$ is determined by the short-run discount factor $\beta \delta$

$$
u^{\prime}\left(c_{t+1}\right)=\delta E_{t+1} V_{1}\left(a_{t+2}, s_{t+2}\right) .
$$

For self $t$, however, the discount factor between periods $t+1$ and $t+2$ is the long-run one, $\delta$, and thus, the values of consumption and assets for self $t$ at

\footnotetext{
${ }^{4}$ For a similar model with iid shocks, Harris and Laibson (2001) prove the existence of equilibrium and provide some general results regarding the continuity and differentiability of the value and policy functions.
} 
period $t+1$ are related as:

$$
u^{\prime}\left(c_{t+1}\right)>\delta E_{t+1} V_{1}\left(a_{t+2}, s_{t+2}\right) .
$$

Given that the marginal utility of consumption is decreasing, from the perspective of self $t$, self $t+1$ overconsumes. The fact that a part of savings is misused by self $t+1$, makes self $t$ to save less, i.e., to act impatiently. On the contrary, if $\beta>1$, self $t$ acts very patiently because, first, she is patient in the short-run (has the short run-discount factor $\beta \delta$, which is higher than the long-run discount factor $\delta$ ) and second, she aims to, at least partially, offset underconsumption of too-patient self $t+1$.

The assumption of quasi-geometric discounting has another important implication: the effective discount factor $\delta_{t+1}$ depends on the agent's wealth. By finding a partial derivative of $\delta_{t+1}$ with respect to $a_{t+1}$ from (10) and omitting the arguments for the sake of compactness, we can write

$$
\frac{\partial \delta_{t+1}}{\partial a_{t+1}}=-\frac{\delta(1-\beta)}{1+r} \cdot \frac{E_{t}\left[u^{\prime \prime} C_{1}^{2}+u^{\prime} C_{11}\right] E_{t}\left[u^{\prime}\right]-E_{t}\left[u^{\prime} C_{1}\right] E_{t}\left[u^{\prime \prime} C_{1}\right]}{E_{t}\left[u^{\prime}\right]^{2}},
$$

where $C_{11} \equiv C_{11}\left(a_{t+1}, s_{t+1}\right)$ is the second order partial derivative of $C$ with respect to the first argument. Consider a non-stochastic steady state of (9) and (10) such that $s_{t}=\bar{s}, c_{t}=\bar{c}$ and $a_{t}=\bar{a}$ for all $t$. By evaluating (11) in the steady state, we get

$$
\frac{\partial \delta(\bar{a}, \bar{s})}{\partial a}=-\frac{\delta(1-\beta)}{1+r} \cdot C_{11}(\bar{a}, \bar{s}) .
$$

Hence, if the consumption function is strictly concave, $C_{11}<0$, then the endogenous discount factor of short-run impatient (patient) consumers is strictly increasing (decreasing) in wealth, at least near the steady state. ${ }^{5}$

The fact that the effective discount factor of agents depends on wealth can play a potentially important role in the model's distributive predictions. We shall recall that under the standard geometric discounting $(\beta=1)$, the model severely underpredicts the wealth of the rich and overpredicts the

\footnotetext{
${ }^{5}$ Carroll and Kimbal (1996) prove analytically that introducing labor income uncertainty into a similar finite-horizon problem with standard geometric discounting, $\beta=1$, and with no restrictions on borrowing induces a concave consumption function. The proof of a parallel result for our setup is beyond the scope of this paper. In our simulations, the consumption function was concave under all parameterization considered.
} 
wealth of the poor agents (see, e.g., Aiyagari, 1994, Quadrini and RíosRull, 1997). Note that the assumption of quasi-geometric discounting can help us to improve on the above shortcoming. Specifically, if $\beta<1$, the rich act more patiently (have a higher discount factor) than the poor. As a result, the difference between the rich and the poor will be larger in the economy with $\beta<1$ than in the one with $\beta=1$, where the rich and the poor are equally patient. In the remainder of the paper, we shall evaluate the effects associated with the assumption of quasi-geometric discounting by using numerical methods.

\section{Quantitative analysis}

In this section, we describe the calibration and solution procedures and discuss the numerical results.

\subsection{Calibration and solution procedures}

The model's period is one year. The long-run discount factor is set at $\delta=$ 0.96. We assume that the production function is Cobb-Douglas, $F(K, N)=$ $K^{\alpha} N^{1-\alpha}$, with a capital share set at $\alpha=0.36$. The depreciation rate of capital $d$ is equal to 0.08 . The debt limit is set at zero, $b=0$.

We assume that the momentary utility function is $u(c)=\frac{c^{1-\gamma}-1}{1-\gamma}$, where $\gamma$ is a constant coefficient of relative risk aversion. As in Aiyagari (1994), we assume that idiosyncratic shocks follow an $A R(1)$ process given by

$$
\log s_{t+1}=\rho \log s_{t}+\sigma\left(1-\rho^{2}\right)^{1 / 2} \varepsilon_{t+1}, \quad \varepsilon_{t+1} \sim N(0,1),
$$

where $\rho \in[0,1]$ is the autocorrelation coefficient, and $\sigma \geq 0$ is the unconditional standard deviation of the variable $\log s_{t}$.

We consider four alternative sets of values of

$$
(\gamma, \rho, \sigma) \in\{(1.0,0.6,0.2),(1.0,0.9,0.2),(3.0,0.9,0.2),(1.0,0.9,0.4)\} .
$$

We assume three alternative values of $\beta \in\{0.8,1.0,1.2\}$, which correspond to the cases of short-run impatience, equal short- and long-run patience and short-run patience, respectively. 
As we argued in section 2.3, the presence of quasi-geometric discounting in the model has two effects. First, the effective discount factor of the agent decreases if $\beta<1$ (increases if $\beta>1$ ) compared to the standard case $\beta=1$. Second, the effective discount factor is not a constant, as in the standard case, but a function of the individual state $\left(a_{t}, s_{t}\right)$. To distinguish between the above effects, we compute the average effective discount factor for each considered economy by using (10). (Such discount factors are denoted by $E\left(\delta_{t} \mid \beta=0.8\right)$ and $E\left(\delta_{t} \mid \beta=1.2\right)$ for the economies with shortrun impatient and short-run patient consumers, respectively.) We then use the average effective discount factors obtained from the models with quasigeometric discounting for calibrating the model with standard geometric discounting. Thus, for each parameterization $(\gamma, \rho, \sigma)$, we report five simulation experiments: three experiments under $\delta=0.96$ and $\beta \in\{0.8,1.0,1.2\}$ and two experiments under $\beta=1.0$ and $\delta \in\left\{E\left(\delta_{t} \mid \beta=0.8\right), E\left(\delta_{t} \mid \beta=1.2\right)\right\}$.

To solve the model, we use an algorithm iterating on the Euler equation. The description of the algorithm is provided in appendix B. ${ }^{6}$ In the benchmark geometric discounting case $(\beta=1)$, the algorithm had no difficulty in computing the solution. Under quasi-geometric discounting $(\beta \neq 1)$, however, the convergence was more costly to achieve. In several experiments, it was necessary to search for a good initial guess for interest rate $r$ and then slowly update the decision rules. Furthermore, the algorithm typically failed to converge when $\beta$ was significantly different from one, specifically, when $\beta$ was out of the range $[0.8,1.2]$. The computational problems described, however, do not appear to be specific to our solution method. ${ }^{7}$

\footnotetext{
${ }^{6}$ Maliar and Maliar (2002) study the convergence properties of this and other Euler equation methods in the context of models with quasi-geometric discounting. The method used in the present paper is shown to yield the same solutions as those obtained by the perturbation method proposed by Krusell, Kuruşçu and Smith (2002).

${ }^{7}$ The difficulties in finding numerical solutions have been reported in other papers on quasi-geometric discounting. Laibson, Repetto and Tobacman (1998) study a finitehorizon model similar to ours and also find that solution can be computed only if $\beta$ is not too low (they use $\beta=0.85$ ). In the context of a deterministic version of the neoclassical growth model with quasi-geometric discounting, Krusell and Smith (2000) argue that numerical problems are related to the fact that in addition to a smooth interior solution, the model has infinitely many discontinuous solutions.
} 


\subsection{Results}

Figure 1 plots the stationary probability distributions of shocks and assets (wealth) in the models under $\beta \in\{0.8,1.0,1.2\}$ and $\delta=0.96$. Two tendencies are evident here. First, the unconditional mean of wealth distribution increases in $\beta$. Second, the fraction of the liquidity-constrained population declines in $\beta$. That is, the agents who are short-run impatient, $\beta=0.8$, are much more likely to be at binding liquidity constraints than those who are short-run patient, $\beta=1.2$.

Table 1 summarizes the statistics on the wealth distribution generated by the model economies. We report two measures of wealth inequality: the Gini coefficient and the percentages of wealth held by different groups of the population. For the sake of comparison, we also provide the corresponding statistics on the U.S. economy.

We must first note that the model with standard geometric discounting $(\beta=1)$ cannot generate the realistic relative degrees of wealth inequality. To be more specific, the poor agents are not so poor and the rich agents are not so rich in the model as they are in the data. For instance, in the model, under $\gamma=1.0, \rho=0.6, \sigma=0.2$ (the first panel in the table), the bottom $40 \%$ of the population holds $17 \%$ of total wealth and the upper $1 \%$ of the population holds $3.1 \%$ of total wealth, whereas in the U.S. economy, these numbers are $2.2 \%$ and $28.2 \%$, respectively. The Gini coefficient reflects the same tendency: it is much lower in the model (0.33) than in the data (0.76). Variations in the parameters $\gamma, \rho, \sigma$ (the remaining three panels in the table) can help to generate a higher concentration of wealth in the model, however, the improvements are not sufficient to account for the data.

We now analyze the case of quasi-geometric discounting. As we mentioned before, the consumption function proved to be concave in our simulations which implies that under the assumption of short-run impatience $\beta<1$ (patience $\beta>1$ ), wealth inequality increases (decreases) in comparison to the one under the equal short- and long-run patience, $\beta=1$. The results in Table 1 make it possible to appreciate the quantitative expressions of these effects. For example, if $\beta=0.8$ and $\gamma=1.0, \rho=0.6, \sigma=0.2$, the model predicts that the wealth holdings of the poorest $40 \%$ of the population are $12.0 \%$ and those of the richest $1 \%$ of the population are $3.5 \%$, (i.e., declines by $29 \%$ and increases by $13 \%$, respectively, compared to the corresponding statistics in the benchmark case $\beta=1.0$ ); similarly, the Gini coefficient rises to 0.39 (i.e., increases by $18 \%$ ). On the contrary, if $\beta=1.2$, the fraction 
Figure 1. The stationary probability distribution of shocks and assets under $\quad \beta=0.8,1.0,1.2$.
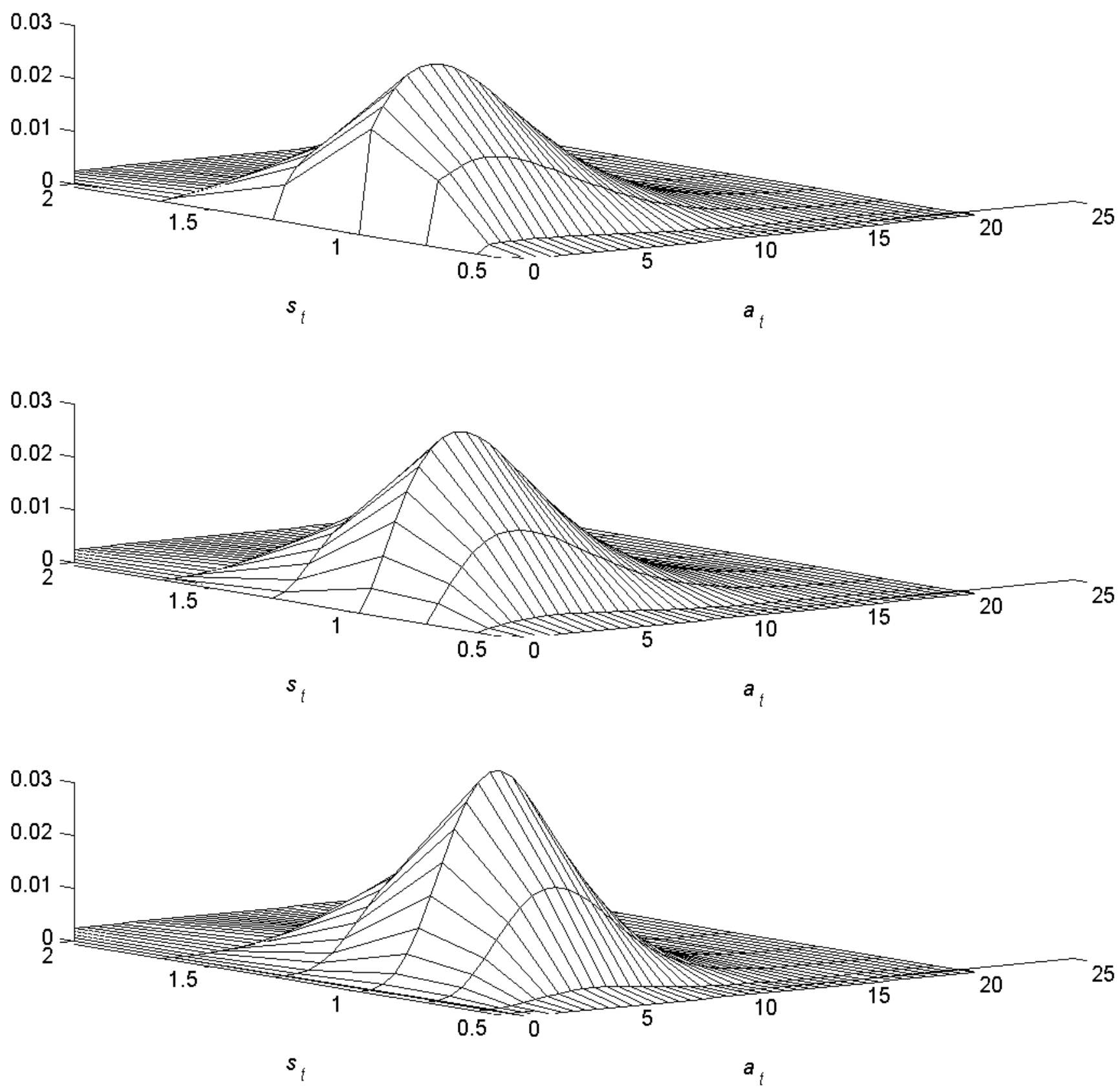

Parameter values: $\alpha=0.36, \gamma=1.0, \delta=0.96, d=0.08, \rho=0.6, \sigma=0.16$. 
Table 1. Selected statistics of the wealth distribution in the U.S. and artificial economies.

\begin{tabular}{|c|c|c|c|c|c|c|c|c|}
\hline & $\beta$ & $\delta$ & Gini & $0-40 \%$ & $\begin{array}{c}80- \\
100 \%\end{array}$ & $\begin{array}{c}90- \\
95 \%\end{array}$ & $\begin{array}{c}95- \\
99 \%\end{array}$ & $\begin{array}{c}99- \\
100 \%\end{array}$ \\
\hline \multicolumn{9}{|l|}{$\gamma=1.0 \quad \rho=0.6 \quad \sigma=0.2$} \\
\hline Quasi-geometric impatient & 0.8 & 0.9600 & 0.39 & 12.0 & 42.2 & 10.4 & 10.4 & 3.5 \\
\hline Geometric, $\delta=E\left(\delta_{t} \mid \beta=0.8\right)$ & 1.0 & 0.9492 & 0.34 & 16.9 & 38.9 & 9.7 & 9.5 & 3.1 \\
\hline Geometric & 1.0 & 0.9600 & 0.33 & 17.0 & 38.6 & 9.5 & 9.5 & 3.1 \\
\hline Geometric, $\delta=E\left(\delta_{t} \mid \beta=1.2\right)$ & 1.0 & 0.9693 & 0.33 & 17.0 & 38.1 & 9.5 & 9.4 & 3.1 \\
\hline Quasi-geometric patient & 1.2 & 0.9600 & 0.23 & 22.8 & 31.2 & 8.1 & 7.5 & 2.3 \\
\hline \multicolumn{9}{|l|}{$\gamma=1.0 \quad \rho=0.9 \quad \sigma=0.2$} \\
\hline Quasi-geometric impatient & 0.8 & 0.9600 & 0.45 & 8.9 & 45.5 & 11.7 & 11.6 & 3.9 \\
\hline Geometric, $\delta=E\left(\delta_{t} \mid \beta=0.8\right)$ & 1.0 & 0.9486 & 0.43 & 10.0 & 43.4 & 11.1 & 10.9 & 3.7 \\
\hline Geometric & 1.0 & 0.9600 & 0.42 & 12.4 & 44.1 & 11.1 & 11.0 & 3.7 \\
\hline Geometric, $\delta=E\left(\delta_{t} \mid \beta=1.2\right)$ & 1.0 & 0.9694 & 0.41 & 12.5 & 43.1 & 11.1 & 11.0 & 3.7 \\
\hline Quasi-geometric patient & 1.2 & 0.9600 & 0.33 & 17.7 & 37.2 & 9.5 & 9.2 & 2.9 \\
\hline \multicolumn{9}{|l|}{$\gamma=3.0 \quad \rho=0.9 \quad \sigma=0.2$} \\
\hline Quasi-geometric impatient & 0.8 & 0.9600 & 0.43 & 10.6 & 43.7 & 111.1 & 11.0 & 3.7 \\
\hline Geometric, $\delta=E\left(\delta_{t} \mid \beta=0.8\right)$ & 1.0 & 0.9475 & 0.40 & 12.1 & 42.2 & 10.6 & 10.6 & 3.5 \\
\hline Geometric & 1.0 & 0.9600 & 0.40 & 13.9 & 42.4 & 10.7 & 10.5 & 3.5 \\
\hline Geometric, $\delta=E\left(\delta_{t} \mid \beta=1.2\right)$ & 1.0 & 0.9693 & 0.39 & 14.0 & 41.5 & 10.7 & 10.5 & 3.4 \\
\hline Quasi-geometric patient & 1.2 & 0.9600 & 0.36 & 15.7 & 39.6 & 10.1 & 9.7 & 3.2 \\
\hline \multicolumn{9}{|l|}{$\gamma=1.0 \quad \rho=0.9 \quad \sigma=0.4$} \\
\hline Quasi-geometric impatient & 0.8 & 0.9600 & 0.47 & 9.7 & " 48.6 & $\begin{array}{ll}12.0 \\
\end{array}$ & 12.6 & "4.4 \\
\hline Geometric, $\delta=E\left(\delta_{t} \mid \beta=0.8\right)$ & 1.0 & 0.9426 & 0.46 & 8.9 & 46.9 & 11.8 & 12.2 & 4.2 \\
\hline Geometric & 1.0 & 0.9600 & 0.45 & 10.4 & 46.6 & 11.7 & 12.0 & 4.1 \\
\hline Geometric, $\delta=E\left(\delta_{t} \mid \beta=1.2\right)$ & 1.0 & 0.9728 & 0.44 & 10.2 & 46.3 & 11.6 & 11.7 & 4.0 \\
\hline Quasi-geometric patient & 1.2 & 0.9600 & 0.41 & 13.3 & 43.2 & 11.0 & 11.0 & 3.6 \\
\hline \multicolumn{3}{|c|}{ U.S. economy ${ }^{(a)}$} & 0.76 & 2.2 & 77.1 & 12.6 & 23.1 & 28.2 \\
\hline
\end{tabular}

${ }^{(a)}$ Source: Quadrini and Ríos-Rule (1997). 
of wealth held by the bottom $40 \%$ of the population is $22.8 \%$ and that held by the top $1 \%$ of the population is $2.3 \%$ (i.e., increases by $34 \%$ and declines by $26 \%$, respectively, compared to the case $\beta=1.0$ ); the Gini coefficient goes down to 0.23 (i.e., declines by $30 \%$ ). The same regularities are observed under the other parameterizations of $(\gamma, \rho, \sigma)$.

As we can see from Table 1, the predictions of the standard model with geometric discounting do not significantly depend on the value of $\delta$ assumed (compare the cases $\delta \in\left\{0.96, E\left(\delta_{t} \mid \beta=0.8\right), E\left(\delta_{t} \mid \beta=1.2\right)\right\}$ ). We therefore conclude that the effect of quasi-geometric discounting on the degrees of wealth inequality in the model comes mostly from the endogenous dependence of the individual effective discount factor on the individual state and not from the differences in the average discount factor across the models.

In Table 2, we include the same statistics on the income distribution, as we previously did for the wealth distribution. As one can see, in the U.S. economy, there is much less dispersion across individuals in income compared with wealth. All model economies are capable of reproducing this regularity, but, again, they dramatically underpredict the degrees of income inequality. The main point to note from the table is that the role of quasi-geometric discounting in the income distribution is quite modest.

The results in Table 3 allow us to quantify the effect of quasi-geometric discounting on the aggregate savings. We can see the tendencies by comparing, e.g., the first three rows of the first panel, $\gamma=1.0, \rho=0.6, \sigma=0.2$. The aggregate capital stock in the economy with quasi-geometric short-run impatient agents $(\beta=0.8)$ is $12.55 \%$ lower than that in the economy with geometric agents $(\beta=1.0)$. The difference between the capital stocks in the models with geometric discounting $(\beta=1.0)$ under $\delta=E\left(\delta_{t} \mid \beta=0.8\right)=0.9492$ and $\delta=0.96$ is even larger: it amounts to $13.26 \%$. We therefore conclude the following: The reduction in the average effective discount factor due to quasigeometric discounting decreases the aggregate savings, but the endogeneity of the effective discount factor actually increases the aggregate savings. Under our benchmark parametrization, the increase in the aggregate savings due to the latter effect is relatively small, $\triangle K^{\beta}=0.71 \%$, however, under other parametrizations, such an increase can be quite sizable. For example, under $\gamma=1.0, \rho=0.9, \sigma=0.4, \beta=0.8$, we have $\triangle K^{\beta}=7.06 \%$. Why does the endogeneity of the effective discount factor raises the aggregate capital stock of the economy with quasi-geometric short-run impatient agents? In section 2.3, we conjectured that with the concave consumption function, the individual effective discount factor of the agent with $\beta<1$ increases in 
Table 2. Selected statistics of the income distribution in the U.S. and artificial economies.

\begin{tabular}{|c|c|c|c|c|c|c|c|c|}
\hline & $\beta$ & $\delta$ & Gini & $0-40 \%$ & $\begin{array}{c}80- \\
100 \%\end{array}$ & $\begin{array}{c}90- \\
95 \%\end{array}$ & $\begin{array}{c}95- \\
99 \% \\
\end{array}$ & $\begin{array}{c}99- \\
100 \%\end{array}$ \\
\hline \multicolumn{9}{|l|}{$\gamma=1.0 \quad \rho=0.6 \quad \sigma=0.2$} \\
\hline Quasi-geometric impatient & 0.8 & 0.9600 & 0.13 & 31.2 & 26.9 & 6.7 & 6.0 & 1.8 \\
\hline Geometric, $\delta=E\left(\delta_{t} \mid \beta=0.8\right)$ & 1.0 & 0.9492 & 0.12 & 31.4 & 26.5 & 6.6 & 5.9 & 1.7 \\
\hline Geometric & 1.0 & 0.9600 & 0.12 & 31.8 & 26.5 & 6.6 & 5.9 & 1.7 \\
\hline Geometric, $\delta=E\left(\delta_{t} \mid \beta=1.2\right)$ & 1.0 & 0.9693 & 0.12 & 32.0 & 26.3 & 6.5 & 5.9 & 1.7 \\
\hline Quasi-geometric patient & 1.2 & 0.9600 & 0.12 & 32.2 & 26.2 & 6.5 & 5.8 & 1.7 \\
\hline \multicolumn{9}{|l|}{$\gamma=1.0 \quad \rho=0.9 \quad \sigma=0.2$} \\
\hline Quasi-geometric impatient & 0.8 & 0.9600 & 0.14 & 30.6 & 27.4 & 6.9 & 6.1 & 1.8 \\
\hline Geometric, $\delta=E\left(\delta_{t} \mid \beta=0.8\right)$ & 1.0 & 0.9486 & 0.14 & 30.7 & 27.3 & 6.9 & 6.1 & 1.7 \\
\hline Geometric & 1.0 & 0.9600 & 0.13 & 31.1 & 27.0 & 6.8 & 6.0 & 1.7 \\
\hline Geometric, $\delta=E\left(\delta_{t} \mid \beta=1.2\right)$ & 1.0 & 0.9694 & 0.13 & 31.4 & 26.8 & 6.8 & 5.9 & 1.7 \\
\hline Quasi-geometric patient & 1.2 & 0.9600 & 0.12 & 31.6 & 26.6 & 6.7 & 5.9 & 1.6 \\
\hline \multicolumn{9}{|l|}{$\gamma=3.0 \quad \rho=0.9 \quad \sigma=0.2$} \\
\hline Quasi-geometric impatient & 0.8 & 0.9600 & 0.13 & 30.9 & 27.2 & 6.9 & 6.0 & 1.7 \\
\hline Geometric, $\delta=E\left(\delta_{t} \mid \beta=0.8\right)$ & 1.0 & 0.9475 & 0.13 & 30.8 & 27.1 & 6.9 & 6.0 & 1.7 \\
\hline Geometric & 1.0 & 0.9600 & 0.13 & 31.4 & 26.8 & 6.8 & 5.9 & 1.7 \\
\hline Geometric, $\delta=E\left(\delta_{t} \mid \beta=1.2\right)$ & 1.0 & 0.9693 & 0.12 & 31.6 & 26.6 & 6.7 & 5.9 & 1.6 \\
\hline Quasi-geometric patient & 1.2 & 0.9600 & 0.12 & 31.6 & 26.6 & 6.7 & 5.9 & 1.6 \\
\hline \multicolumn{9}{|l|}{$\gamma=1.0 \quad \rho=0.9 \quad \sigma=0.4$} \\
\hline Quasi-geometric impatient & 0.8 & 0.9600 & 0.23 & 24.8 & 33.2 & 8.4 & 8.0 & 2.3 \\
\hline Geometric, $\delta=E\left(\delta_{t} \mid \beta=0.8\right)$ & 1.0 & 0.9426 & 0.23 & 24.6 & 33.5 & 8.4 & 8.1 & 2.3 \\
\hline Geometric & 1.0 & 0.9600 & 0.23 & 24.9 & 33.3 & 8.3 & 8.1 & 2.3 \\
\hline Geometric, $\delta=E\left(\delta_{t} \mid \beta=1.2\right)$ & 1.0 & 0.9728 & 0.23 & 25.1 & 33.1 & 8.3 & 8.1 & 2.3 \\
\hline Quasi-geometric patient & 1.2 & 0.9600 & 0.23 & 25.1 & 33.2 & 8.3 & 8.0 & 2.3 \\
\hline \multicolumn{3}{|c|}{ U.S. economy ${ }^{(a)}$} & 0.51 & 10.3 & 53.6 & 10.7 & 13.5 & 14.1 \\
\hline
\end{tabular}

${ }^{(a)}$ Source: Quadrini and Ríos-Rule (1997). 
Table 3. Equilibrium interest rate and aggregate capital in the artificial economies.

\begin{tabular}{|c|c|c|c|c|c|c|c|c|}
\hline & $\beta$ & $\delta$ & $r, \%$ & $K$ & $\Delta K, \%$ & $\Delta K^{\beta}, \%$ & $K^{s s}$ & $P S, \%$ \\
\hline \multicolumn{9}{|l|}{$\gamma=1.0 \quad \rho=0.6 \quad \sigma=0.2$} \\
\hline Quasi-geometric impatient & 0.8 & 0.9600 & 5.05 & 44.88 & -12.55 & 0.71 & 4.79 & 1.88 \\
\hline Geometric, $\delta=E\left(\delta_{t} \mid \beta=0.8\right)$ & 1.0 & 0.9492 & 5.12 & 4.84 & -13.26 & & 4.71 & 2.76 \\
\hline Geometric & 1.0 & 0.9600 & 3.98 & 5.58 & 0.0 & & 5.45 & 2.38 \\
\hline Geometric, $\delta=E\left(\delta_{t} \mid \beta=1.2\right)$ & 1.0 & 0.9693 & 3.02 & 6.36 & 13.98 & & 6.23 & 2.09 \\
\hline Quasi-geometric patient & 1.2 & 0.9600 & 3.09 & 6.30 & 12.90 & -1.08 & 5.97 & 5.53 \\
\hline \multicolumn{9}{|l|}{$\gamma=1.0 \quad \rho=0.9 \quad \sigma=0.2$} \\
\hline Quasi-geometric impatient & 0.8 & 0.9600 & 4.97 & 4.93 & -12.43 & 1.42 & 4.79 & 2.92 \\
\hline Geometric, $\delta=E\left(\delta_{t} \mid \beta=0.8\right)$ & 1.0 & 0.9486 & 5.11 & 4.85 & -13.85 & & 4.67 & 3.85 \\
\hline Geometric & 1.0 & 0.9600 & 3.91 & 5.63 & 0.0 & & 5.45 & 3.30 \\
\hline Geometric, $\delta=E\left(\delta_{t} \mid \beta=1.2\right)$ & 1.0 & 0.9694 & 2.93 & 6.44 & 14.39 & & 6.24 & 3.21 \\
\hline Quasi-geometric patient & 1.2 & 0.9600 & 3.05 & 6.33 & 12.43 & -1.96 & 5.97 & 6.03 \\
\hline \multicolumn{9}{|l|}{$\gamma=3.0 \quad \rho=0.9 \quad \sigma=0.2$} \\
\hline Quasi-geometric impatient & 0.8 & 0.9600 & 4.21 & 5.42 & -11.29 & 3.44 & 4.79 & 13.15 \\
\hline Geometric, $\delta=E\left(\delta_{t} \mid \beta=0.8\right)$ & 1.0 & 0.9475 & 4.52 & 5.21 & -14.73 & & 4.61 & 13.01 \\
\hline Geometric & 1.0 & 0.9600 & 3.31 & 6.11 & 0.0 & & 5.45 & 12.11 \\
\hline Geometric, $\delta=E\left(\delta_{t} \mid \beta=1.2\right)$ & 1.0 & 0.9693 & 2.44 & 6.92 & 13.26 & & 6.23 & 11.08 \\
\hline Quasi-geometric patient & 1.2 & 0.9600 & 2.63 & 6.73 & 10.15 & -3.11 & 5.97 & 12.73 \\
\hline \multicolumn{9}{|l|}{$\gamma=1.0 \quad \rho=0.9 \quad \sigma=0.4$} \\
\hline Quasi-geometric impatient & 0.8 & 0.9600 & 4.40 & 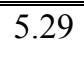 & -13.14 & 7.06 & 4.79 & 10.44 \\
\hline Geometric, $\delta=E\left(\delta_{t} \mid \beta=0.8\right)$ & 1.0 & 0.9426 & 5.09 & 4.86 & -20.20 & & 4.33 & 12.24 \\
\hline Geometric & 1.0 & 0.9600 & 3.33 & 6.09 & 0.0 & & 5.45 & 11.74 \\
\hline Geometric, $\delta=E\left(\delta_{t} \mid \beta=1.2\right)$ & 1.0 & 0.9728 & 2.09 & 7.30 & 19.87 & & 6.57 & 11.11 \\
\hline Quasi-geometric patient & 1.2 & 0.9600 & 2.38 & 6.98 & 14.61 & -5.26 & 5.97 & 16.92 \\
\hline
\end{tabular}

Notes: Statistic $\Delta K$ is the percentage difference between the capital stock in the raw and the one in the geometric-discounting case $\beta=1.0$ and $\delta=0.96 ; \Delta K^{\beta}$ is the difference between $\Delta K$ for the quasi-geometricdiscounting case with $\beta=0.8$ and $\delta=0.96(\beta=1.2$ and $\delta=0.96)$ and $\Delta K$ for the geometric-discounting case with $\beta=1.0$ and $\delta=E\left(\delta_{t} \mid \beta=0.8\right)\left(\beta=1.0\right.$ and $\left.\delta=E\left(\delta_{t} \mid \beta=1.2\right)\right) ; K^{s s}$ is the capital stock in the steady state of the deterministic version of the model; and $P S, \%$ is precautionary savings, which are defined as the percentage difference between the capital stocks in the stochastic and deterministic models. 
wealth. As a result, the savings rate of the rich increases, and that of the poor decreases. Since the aggregate amount of savings is mainly determined by the rich, this pushes the aggregate capital stock up. With quasi-geometric short-run patient agents $(\beta=1.2)$, all the tendencies are reversed.

In Table 3, we also report the amount of precautionary savings, $P S, \%$, which are defined as the percentage difference between the capital stock in the stochastic economy, $K$, and the one in the associated deterministic economy, $K^{s s}$. The main finding here is that the difference in precautionary savings across the models in each panel is relatively small. This is in contrast to the result of Laibson, Repetto and Tobacman (1998) that under the low values of $\beta$ (specifically, they use $\beta=0.85$ ), the presence of quasi-geometric discounting leads to the missing precautionary savings effect. ${ }^{8}$ The discrepancy between the results of Laibson, Repetto and Tobacman (1998) and ours is explained by the fact that in their model, the interest rate is given exogenously, whereas, in our model, it is determined endogenously. In a general equilibrium setup like ours, the agents' willingness to save more (less) drives the interest rate down (up), which, in turn, decreases (increases) the incentives to save. Precisely this mitigates the effect of quasi-geometric discounting on precautionary savings.

\section{Concluding remarks}

The standard one-sector growth model with a large number of agents subject to uninsured idiosyncratic shocks predicts substantially less wealth inequality than what is observed in the data. One way of generating more skewness in the distribution of wealth is to assume that agents differ in patience (discount factors), e.g., Krusell and Smith (1995, 1998) and Carroll (2000). In the paper, we argue that the introduction of quasi-geometric discounting can have the same effect on the equilibrium as postulating heterogeneity in the discount factors. This is because the effective discount factor becomes an endogenous state-dependent variable. In particular, if agents are short-run impatient, then the effective discount factor increases in wealth, which accentuates the differences between the saving rates of rich and poor agents.

\footnotetext{
${ }^{8}$ The empirical findings about the importance of a precautionary savings motive are mixed. For example, Carroll (1994), Carroll and Samwick (1997) find strong evidence of precautionary savings, while Dynan (1993), Guiso, Jappelli and Terlizzese (1992) report the missing precautionary savings effect.
} 
The consequence is that the model with quasi-geometric short-run impatient agents produces a larger dispersion of wealth than does the standard geometric-discounting setup. We evaluate the effects associated with quasigeometric discounting in a calibrated version of the model. We find that such effects are quantitatively significant but not sufficient in order the model can account for the degrees of wealth and income inequalities in the data.

\section{References}

[1] Aiyagari, R., 1994, Uninsured idiosyncratic risk and aggregate saving, Quarterly Journal of Economics 109(3), 659-684.

[2] Barro, R., 1999, Ramsey meets Laibson in the neoclassical growth model, Quarterly Journal of Economics 114 (4), 1125-1152.

[3] Carroll, C., 1994, How does future income affect current consumption? Quarterly Journal of Economics 109, 111-148.

[4] Carroll, C., 2000, Requiem for the representative consumer? Aggregate implications of microeconomic consumption behavior, Manuscript.

[5] Carroll, C. and M. Kimbal, 1996, On the concavity of the consumption function, Econometrica 64(4), 981-992.

[6] Carroll, C. and A. Samwick, 1998, How important is precautionary saving? Review of Economics and Statistics 80, 410-419.

[7] Dynan, K., 1993, How prudent are consumers?, Journal of Political Economy 101(6), 1104-1113.

[8] Hall, R., 1998, Self-control and saving for retirement, Comments and discussion, Brookings Papers on Economic Activity 1, 173-177.

[9] Harris, C. and D. Laibson, 2001, Dynamic choices of hyperbolic consumers, Econometrica 69 (4), 935-959.

[10] Huggett, M., 1993, The risk-free rate in heterogeneous-agent incompleteinsurance economies, Journal of Economic Dynamics and Control 17, 953-969. 
[11] Huggett, M., 1997, The one-sector growth model with idiosyncratic shocks: Steady states and dynamics, Journal of Monetary Economics 39, 385-403.

[12] Guiso, L., Jappelli, T. and D.Telizzese, 1992, Earning uncertainty and precautionary saving, Journal of Monetary Economics 30, 307-337.

[13] Krusell, P. and A. Smith, 1995, Income and wealth heterogeneity in the macroeconomy, Working paper 399 (Rochester Center for Economic Research, Rochester, NY).

[14] Krusell, P. and A. Smith, 1998, Income and wealth heterogeneity in the macroeconomy, Journal of Political Economy 106(5), 868-896.

[15] Krusell, P. and A. Smith, 2000, Consumption-savings decisions with quasi-geometric discounting, CEPR discussion paper no. 2651.

[16] Krusell, P. and A. Smith, 2003, Consumption-savings decisions with quasi-geometric discounting, Econometrica 71, 365-375.

[17] Krusell, P., B. Kuruşçu and A. Smith, 2002, Equilibrium welfare and government policy with quasi-geometric discounting, Journal of Economic Theory 105, 42-72.

[18] Laibson, D., 1997, Golden eggs and hyperbolic discounting, Quarterly Journal of Economics 112(2), 443-477.

[19] Laibson, D., A. Repetto, and J. Tobacman, 1998, Self-control and saving for retirement, Brookings Papers on Economic Activity 1, 91-172.

[20] Luttmer, E., and T. Mariotti, 2002, Subjective discounting in an exchange economy, forthcoming in Journal of Political Economy.

[21] Maliar, L. and S. Maliar, 2002, Solving the neoclassical growth model with quasi-geometric discounting: non-linear Euler-equation methods, Manuscript, http://merlin.fae.ua.es/maliarl.

[22] Phelps, E. and R. Pollak, 1968, On second-best national saving and game-equilibrium growth, Review of Economic Studies 35, 185-199.

[23] Pollak, R., 1968, Consistent planning, Review of Economic Studies, 35, 201-208. 
[24] Quadrini, V. and V. Ríos-Rull, 1997, Understanding the US distribution of wealth, Federal Reserve Bank of Minneapolis Quarterly Review 21, $22-36$.

[25] Rios-Rull, V., 1999, "Computation of equilibria in heterogeneous agent models", in: Marimon, R., Scott, A., eds., Computational Methods for Study of Dynamic Economies, Oxford University Press, New York.

[26] Strotz, R., 1955-1956, Myopia and inconsistency in dynamic utility maximization, Review of Economic Studies 23, 165-180.

\section{Appendices}

Appendix A contains the proof to Lemma 1. Appendix B presents a description of the computational algorithm.

\subsection{Appendix A}

Proof to Lemma 1. Denote $u\left(c_{t}\right)=u\left((1+r) a_{t}+w s_{t}-a_{t+1}\right) \equiv u\left(a_{t}, a_{t+1}\right)$.

We first prove that the asset function $A\left(a_{t}, s_{t}\right)$ is strictly increasing in $a_{t}$. For any two levels of current wealth $a_{t}^{1}$ and $a_{t}^{2}$ and the corresponding next period's wealth $a_{t+1}^{1}=A\left(a_{t}^{1}, s_{t}\right)$ and $a_{t+1}^{2}=A\left(a_{t}^{2}, s_{t}\right)$, we have

$$
\begin{aligned}
& u\left(a_{t}^{1}, a_{t+1}^{1}\right)+\beta \delta E_{t}\left[V\left(a_{t+1}^{1}, s_{t+1}\right)\right]>u\left(a_{t}^{1}, a_{t+1}^{2}\right)+\beta \delta E_{t}\left[V\left(a_{t+1}^{2}, s_{t+1}\right)\right] \\
& u\left(a_{t}^{2}, a_{t+1}^{2}\right)+\beta \delta E_{t}\left[V\left(a_{t+1}^{2}, s_{t+1}\right)\right]>u\left(a_{t}^{2}, a_{t+1}^{1}\right)+\beta \delta E_{t}\left[V\left(a_{t+1}^{1}, s_{t+1}\right)\right] .
\end{aligned}
$$

On adding up these equations and rearranging the terms, we obtain

$$
u\left(a_{t}^{1}, a_{t+1}^{1}\right)-u\left(a_{t}^{1}, a_{t+1}^{2}\right)>u\left(a_{t}^{2}, a_{t+1}^{1}\right)-u\left(a_{t}^{2}, a_{t+1}^{2}\right) .
$$

The strict concavity of the utility function implies that if $a_{t}^{1}>a_{t}^{2}$, then $a_{t+1}^{1}>a_{t+1}^{2}$, i.e., that $A\left(a_{t}, s_{t}\right)$ is strictly increasing in $a_{t}$.

In order to prove that the consumption function $C\left(a_{t}, s_{t}\right)$ is strictly increasing in $a_{t}$, we use the results that the optimal value function $W\left(a_{t}, s_{t}\right)$ is strictly increasing and strictly concave in $a_{t}$.

The fact that $W$ is strictly increasing in $a_{t}$ follows from the assumption of the strictly increasing utility function $u$ and by the definition of

$$
W\left(a_{t}, s_{t}\right)=\max _{a_{t+1}}\left\{u\left((1+r) a_{t}+w s_{t}-a_{t+1}\right)+\beta \delta E_{t}\left[V\left(a_{t+1}, s_{t+1}\right)\right]\right\} .
$$


The strict concavity of $W$ can be shown as follows: Fix a sequence of realizations for shocks $\left(s_{t}, s_{t+1}, \ldots\right) \in \mathcal{S}$. Consider $a_{t}^{1}$ and $a_{t}^{2}$ such that $a_{t}^{1}>a_{t}^{2}$. By using the asset function $A\left(a_{t}, s_{t}\right)$ iteratively, we find the corresponding optimal sequences for assets $\left(a_{t}^{1}, a_{t+1}^{1}, \ldots\right) \in \mathcal{A}$ and $\left(a_{t}^{2}, a_{t+1}^{2}, \ldots\right) \in \mathcal{A}$. The fact that $A\left(a_{t}, s_{t}\right)$ is strictly increasing in $a_{t}$ implies that $a_{\tau}^{1}>a_{\tau}^{2}$ for all $\tau \geq t$.

Consider the sequence $\left(\lambda a_{t}^{1}+(1-\lambda) a_{t}^{2}, \lambda a_{t+1}^{1}+(1-\lambda) a_{t+1}^{2}, \ldots\right) \in \mathcal{A}$, where $\lambda \in[0,1]$. The strict concavity of $W$ follows from the strict concavity of the utility function $u$

$$
\begin{gathered}
W\left(\lambda a_{t}^{1}+(1-\lambda) a_{t}^{2}, s_{t}\right)= \\
u\left(\lambda a_{t}^{1}+(1-\lambda) a_{t}^{2}, \lambda a_{t+1}^{1}+(1-\lambda) a_{t+1}^{2}\right)+ \\
E_{t} \sum_{\tau=t}^{\infty} \beta \delta^{\tau+1-t} u\left(\lambda a_{\tau}^{1}+(1-\lambda) a_{\tau}^{2}, \lambda a_{\tau+1}^{1}+(1-\lambda) a_{\tau+1}^{2}\right)> \\
>\lambda\left[u\left(a_{t}^{1}, a_{t+1}^{1}\right)+E_{t} \sum_{\tau=t}^{\infty} \beta \delta^{\tau+1-t} u\left(a_{\tau}^{1}, a_{\tau+1}^{1}\right)\right]+ \\
(1-\lambda)\left[u\left(a_{t}^{2}, a_{t+1}^{2}\right)+E_{t} \sum_{\tau=t}^{\infty} \beta \delta^{\tau+1-t} u\left(a_{\tau}^{2}, a_{\tau+1}^{2}\right)\right]= \\
\lambda W\left(a_{t}^{1}, s_{t}\right)+(1-\lambda) W\left(a_{t}^{2}, s_{t}\right) .
\end{gathered}
$$

To complete the proof, we find the derivative of $W$ from $(4)-(6)$

$$
W_{1}\left(a_{t}, s_{t}\right)=u^{\prime}\left(C\left(a_{t}, s_{t}\right)\right)(1+r) .
$$

The fact that $W$ is strictly increasing and strictly concave in $a_{t}$ implies that $C\left(a_{t}, s_{t}\right)$ is strictly increasing in $a_{t}$. 


\subsection{Appendix B}

To compute the equilibrium, we approximate the autoregressive process for the shocks by a seven-state Markov chain, as in Aiyagari (1994). For each state $s \in\left\{s_{1}, \ldots, s_{7}\right\}$, we parametrize the asset demand by a function of the current asset holdings. ${ }^{9}$ The grid for asset holdings consists of 100 equally spaced points in the range $\left[a_{\min }, a_{\max }\right]$, where $a_{\min } \equiv b=0$ and $a_{\max }$ is the maximum sustainable capital stock (i.e., the solution to $f(a)=d a$ ). To evaluate the asset function outside the grid, we use cubic polynomial interpolation.

We employ the algorithm, which iterates on the Euler equation. By substituting consumption from the Euler equation (8) in budget constraint (6), we obtain

$$
\begin{gathered}
a^{\prime}=(1+r) a+w s- \\
-\left\{\delta \sum_{s \in\left\{s_{1}, \ldots, s_{7}\right\}}\left[\frac{\left(1+r-(1-\beta) C_{1}\left(A(a, s), s^{\prime}\right)\right)}{\left(A(a, s)(1+r)+w s^{\prime}-A\left(A(a, s), s^{\prime}\right)\right)^{\gamma}}\right] \cdot \operatorname{Prob}\left(s^{\prime} \mid s\right)\right\}^{-1 / \gamma}
\end{gathered}
$$

Consequently, we implement the following iterative procedure:

- Step 1. Fix some asset function on the grid, $A(a, s)$ and compute the corresponding consumption function $C(a, s)$ from budget constraint (6).

- Step 2. Use the decision rules to calculate the right side of the Euler equation (13) in each point on the grid. The left side of the Euler equation will be the new asset function, $\tilde{A}(a, s)$.

- Step 3. Compute the asset function for next iteration $\widetilde{\widetilde{A}}(a, s)$ by using the updating:

$$
\widetilde{\widetilde{A}}(a, s)=\eta \tilde{A}(a, s)+(1-\eta) A(a, s), \quad \eta \in(0,1] .
$$

\footnotetext{
${ }^{9}$ The borrowing restriction on assets used in our paper, $a^{\prime} \geq 0$, is not equivalent to the one in Aiyagari (1994). In the latter paper, the restriction is imposed on total resources. These are restricted to being no lower than the wage corresponding to an interest rate equal to the time preference rate (the highest possible interest rate under $\beta=1$ ). Such restriction on the total resources would not be appropriate if discounting is quasi-geometric $(\beta \neq 1)$, as the equilibrium interest rate can be either higher and or lower than the time preference rate.
} 
For each point, such that $\widetilde{\widetilde{A}}(a, s)$ does not belong to $\left[a_{\min }, a_{\max }\right]$, set $\widetilde{\widetilde{A}}(a, s)$ at the corresponding boundary value.

- Iterate on Steps $1-3$ until $\widetilde{\widetilde{A}}(a, s)=A(a, s)$ with a given precision.

In the stochastic case, we compute the interest rate and wage corresponding to the given asset function $A(a, s)$ by using invariant probability distribution of shocks and assets, as described in Rios-Rull (1999):

$$
\operatorname{Prob}\left(a^{\prime}, s^{\prime}\right)=\sum_{s \in\left\{s_{1}, \ldots, s_{7}\right\}} \operatorname{Prob}\left(A^{-1}\left(a^{\prime}, s\right), s\right) \cdot \operatorname{Prob}\left(s^{\prime} \mid s\right)
$$

where $A^{-1}\left(a^{\prime}, s\right)=\left\{a, a^{\prime}=A(a, s)\right\}$ is the inverse of the asset function. To compute the interest rate and wage corresponding to the given asset function in the deterministic economy, we solve for a fixed point satisfying the property $A\left(a^{*}, 1\right)=a^{*}$.

Finally, to solve for the equilibrium (the stochastic steady state), we use the bisection method as in Aiyagari (1994). 